\title{
STRUCTURE OF GLOBULAR CLUSTERS
}

\author{
G. MEYLAN \\ European Southern Observatory, Karl-Schwarzschild-Strasse 2, \\ D-85748 Garching bei München, Germany
}

AND

G.A. DRUKIER

Dept. of Astronomy, Indiana University, 319 Swain West, Bloomington, IN, 47401, USA

\section{Overall Radial Structure (G. Meylan)}

There was a time, more than a century ago, when Astronomy \& Astrophysics was not a European Journal yet, but still an American one. In its Vol. XII, Bailey (1893) published, from photographic observations of two globular clusters (GCs), $\omega$ Centauri and 47 Tucanae, what were probably the first extensive star counts, which represent the oldest observational constraint for the study of the structure of GCs. Bailey's counts, together with some new material concerning other clusters were used by Pickering (1897) in the first important comparisons between observed and theoretical profiles in order to study the radial distribution of stars in clusters.

Early in the $20^{\text {th }}$ century, some parallels were drawn between a molecular gas and star clusters. Plummer (1911), Eddington (1916), and Jeans (1916), pursued the search for a physical basis on which the distribution of stars in GCs could be established. Ambartsumian (1938), Spitzer (1940), and Chandrasekhar (1942) investigated the consequences of stellar encounters.

The next burst of fundamental papers took place in the early sixties, with the contributions by Michie (1963), and King (1966) among others. King (1966) provided, for the first time, a grid of models with different concentrations $c=\log \left(r_{t} / r_{c}\right)\left(r_{t}\right.$ and $r_{c}$ are the tidal and core radii, respectively) that approximately incorporated the three most important elements governing GC structure: dynamical equilibrium, two-body relaxation, and tidal truncation. Von Hoerner (1957), Oort \& van Herk (1959), Dickens \& Woolley (1967), and Gunn \& Griffin (1979) initiated the modern interplay of observation and model-building that still continues today.

The structure of a GC is defined at each moment by a distribution function in a phase space with 7 dimensions (positions, velocities, and time). Unfortunately, the numerical study of such a general form is intractable. It is necessary to make some simplifying hypotheses, e.g., in considering separately the problems of structure and evolution. In reality, structure and evolution cannot be dissociated: they are intimately linked, each one determining the other. Hénon (1961) made the first attempt to solve the structure and evolution equations simultaneously and had a first glance at what was to become the Holy Grail of GC dynamics: core collapse. See Meylan \& Heggie (1997) for a more complete review.

\section{Parametric and non-parametric approaches (G. Meylan)}

During the last decades, the most common approach in the comparison between observed and theoretical radial density and velocity dispersion profiles has been based on the use of Jeans' theorem for general solutions of the collisionless Boltzmann equation. Many different kinds of models may be constructed with this parametric approach. In the first place, there is considerable freedom of choice over which integrals to include. In the second place, one is free to choose the functional dependence of these integrals, i.e., the analytic form of the distribution function (see, e.g., Binney \& Tremaine 1987). 
One of the most recent such parametric studies used the mean radial velocities of 469 individual stars (Mayor et al. 1997) located in the galactic GC $\omega$ Centauri $\equiv$ NGC 5139 to derive its velocity dispersion profile (Meylan et al. 1995). A simultaneous fit of these radial velocities and of the surface brightness profile to a multi-mass King-Michie dynamical model provides mean estimates of the total mass equal to $M_{\text {tot }}=5.110^{6} M_{\odot}$, with a corresponding mean mass-to-light ratio $M / L_{V}=$ 4.1. The present results emphasize the fact that $\omega$ Centauri is not only the brightest but also, by far, the most massive galactic GC (Meylan et al. 1995).

Since the early nineties, it has been recognized that results can be strongly biased by the assumed functional form. Solutions are not unique, since different amounts of anisotropy and different potentials can mimic identical surface brightness profiles. The aim of such a non-parametric approach is to infer the gravitational potential and the distribution function given observations of the surface density and velocity dispersion profiles of a tracer population (Merritt 1996).

The same mean radial velocities of 469 individual stars (Mayor et al. 1997) have been used by Merritt et al. (1997) in a non-parametric study of $\omega$ Centauri. Their principal conclusions are that the rotational velocity field in $\omega$ Centauri is consistent with axisymmetry, once a correction is made for "perspective rotation" resulting from the cluster's proper motion. The rotation is strongly noncylindrical, with a peak rotation speed of $7.9_{-2.5}^{+2.4} \mathrm{~km} \mathrm{~s}^{-1}(95 \%)$ at a distance of $\sim 11 \mathrm{pc}$ from the cluster center in the equatorial plane. The rotation is approximately solid-body at small radii; at large radii, the available data do not strongly constrain the form of the rotational velocity field. The central velocity dispersion parallel to the meridional plane is $\sigma(0,0)=17_{-2.6 \mathrm{j}}^{+2.1} \mathrm{~km} \mathrm{~s}^{-1}$. There is no evidence for significant anisotropy anywhere in $\omega$ Centauri. Thus, this cluster can reasonably be described as an "isotropic oblate rotator." The gravitational potential and mass distribution in $\omega$ Centauri are consistent with the predictions of a model in which the mass is distributed in the same way as the bright stars. The derived two-integral distribution function $f\left(E, L_{z}\right)$ for the stars in $\omega$ Centauri is fully consistent with the data.

These non-parametric results are consistent with the previous parametric ones, and illustrate the need for large samples of radial velocities (a few thousands) in order to take full advantage of this new approach. Samples of about 4,000 stars are announced by Seitzer et al. and by Côté et al. (private communications).

\section{Mayall II $\equiv$ G1, a Globular Cluster in M31 (G. Meylan)}

For distant GCs, usual simple approaches remain the only solution for mass estimates. E.g., in the case of Mayall II $\equiv \mathrm{G} 1$, two observational constraints exist: (i) the surface brightness profile from HST/WFPC2 data by Jablonka et al. (1997) providing the core radius $r_{c}=0.24^{\prime \prime}=0.9 \mathrm{pc}$, the tidal radius $r_{t} \simeq 54^{\prime \prime}=200 \mathrm{pc}$, and the concentration $c=\log \left(r_{t} / r_{c}\right) \simeq 2.35$; (ii) the central velocity dispersion from KECK/HIRES data by Djorgovski et al. (1997), with an observed velocity dispersion $\sigma_{o b s}=25.1 \mathrm{~km} \mathrm{~s}^{-1}$ and an aperture corrected core velocity dispersion $\sigma_{p}(0)=27.3$ $\mathrm{kms}^{-1}$.

King-model and Virial mass approaches (Illingworth 1976) provide two first estimates of the total mass of this GC. First, since King mass $=\rho_{c} r_{c}^{3} \mu=167 r_{c} \mu \sigma_{p}(0)^{2}$, with

$$
\begin{aligned}
& \text { - } r_{c}=0.9 \mathrm{pc} \text {, } \\
& \text { - } \mu=125.7 \text { for } \mathrm{c}=2.35 \text { (King 1966), } \\
& \text { - } \sigma_{p}(0)=27.3 \mathrm{~km} \mathrm{~s}^{-1},
\end{aligned}
$$

the total King-model mass $M=14 \times 10^{6} M_{\odot}$ and the corresponding mass-to-light ratio $M / L \simeq$ 10. Second, since Virial mass $=670 r_{h} \sigma_{p}(0)^{2}$, with

- $r_{h}=13.7 \mathrm{pc}$ and

- $\sigma_{p}(0)=27.3 \mathrm{~km} \mathrm{~s}^{-1}$,

the total Virial mass $M=6.8 \times 10^{6} M_{\odot}$ and the corresponding mass-to-light ratio $M / L \simeq 4.9$.

By using a King-Michie model (Gunn \& Griffin 1979, Meylan 1987) fitted simultaneously to the surface brightness profile from HST/WFPC2 and the central velocity dispersion value from KECK/HIRES, and recovering the total integrated absolute luminosity $M_{V}=-10.55 \mathrm{mag}$, a grid of about 10,000 models has been calculated for a wide range of values of each parameter. Parameter values for two rather different - equally good - models are presented in Table 1 where $x$ is the mass function exponent (equal to 1.35 in Salpeter's case), $M_{n s}$ and $M_{w d}$ are the fractions of the total mass in neutron stars and in white dwarfs, respectively, conc is the concentration $c=\log \left(r_{t} / r_{c}\right)$ 
determined from the surface brightness profile, $r_{a}$ is the anisotropy radius, $M_{t o t}$ the cluster total mass, and $M / L_{V}$ the cluster mass-luminosity ratio. Good models populate very specific (although non unique) areas of the parameter space.

TABLE 1. Mayal II $\equiv G 1$ : two rather different models producing equally good fits of the surface brightness profile and matching the total integrated absolute luminosity $M_{V}=-10.55 \mathrm{mag}$.

\begin{tabular}{ccccccc}
\hline$x$ & $\begin{array}{c}M_{n s} \\
\%\end{array}$ & $\begin{array}{c}M_{w d} \\
\%\end{array}$ & $\begin{array}{c}\text { conc } \\
\log \left(r_{t} / r_{c}\right)\end{array}$ & $r_{a} / r_{c}$ & $\begin{array}{c}M_{\text {tot }} \\
{\left[10^{6} M_{\odot}\right]}\end{array}$ & $M / L_{V}$ \\
\hline 0.75 & 0.14 & 16 & 2.36 & 15 & 10.1 & 7.1 \\
2.00 & 0.06 & 7.3 & 2.43 & 25 & 18.7 & 13.4 \\
\hline
\end{tabular}

Obviously, the lack of a velocity dispersion profile prevents the process of narrowing down the ranges of parameters. Nevertheless, we can reach the following conclusions about Mayall II $\equiv$ G1: - All mass estimates (King, Virial, and King-Michie) give G1 more than twice as massive as $\omega$ Centauri, the most massive galactic GC; this does not come as a surprise given the fact that their total integrated absolute luminosities are $M_{V}=-10.55 \mathrm{mag}$ for G1 and -10.07 mag for $\omega$ Centauri. - With $c=\log \left(r_{t} / r_{c}\right)=2.35, \mathrm{G} 1$ is significantly more concentrated than $\omega$ Centauri, which has $c$ $=1.24$, and even more concentrated than 47 Tucanae, which has $c=2.04$.

- Although G1 is the heaviest of the weighted GCs, it would be a hasty conclusion to claim that G1, even more than $\omega$ Centauri, is a kind of transition step between GCs and dwarf elliptical galaxies. When considering the positions of G1 in the different diagrams defined by Kormendy (1985) (viz., central surface brightness vs. core radius, central surface brightness vs. absolute magnitude, central velocity dispersion vs. core radius, core radius vs. absolute magnitude), $\mathrm{G} 1$ appears always close to the sequence defined by GCs, and away from the sequences defined by elliptical galaxies, bulges, and dwarf spheroidal galaxies. Consequently, Mayall II $\equiv \mathrm{G} 1$ is a genuine, bright and massive GC.

\section{Central Density Cusp from HST Observations (G. Meylan)}

The whole concept of core collapse, linked to the gravothermal instability which may develop in a gravitational system because of its negative specific heat, was investigated in the early sixties (see Lynden-Bell \& Wood 1968). Fokker-Planck and conducting-gas-sphere evolutionary models have now been computed well into core collapse and beyond, leading to the discovery of post-collapse oscillations (Makino 1996). From an observational point of view, the presence of core collapse could be betrayed by the presence of power-law cusps in both the density and velocity dispersion profiles (see Meylan \& Heggie 1997 for a review).

Sosin \& King (1997) investigated the presence of a possible cusp in the density profile of the prototype of the collapsed-core GCs, viz, M15. They made star counts on the sharpest images of this cluster, obtained by HST/FOC with filter F480LP $\equiv$ FOC $V$ with a pixel size $0.014^{\prime \prime} /$ pix. They counted:

- 577 stars with $18.25 \leq V \leq 19.75$, i.e., with mass $\sim 0.8 M_{\odot}$,

- 1222 stars with $19.75 \leq V \leq 21.25$, i.e., with mass $0.67 \leq M \leq 0.77 M_{\odot}$, and

- 539 stars with $21.25 \leq V \leq 22.75$, i.e., with mass $0.55 \leq M \leq 0.67 M_{\odot}$.

They determined a $\operatorname{logarithmic}$ cusp slope $d \log \sigma / d \log r=-0.70 \pm 0.05$, between $0.3^{\prime \prime}$ and $10^{\prime \prime}$, for stars with $M \sim 0.8 M_{\odot}$, and $d \log \sigma / d \log r=-0.56 \pm 0.05$, between $2.0^{\prime \prime}$ and $10^{\prime \prime}$, for stars with $M$ $\sim 0.7 M_{\odot}$. They set an upper limit of $1.5^{\prime \prime}$ on the size of any possible constant-surface-density core. Among the galactic GCS, M15 displays one of the clearest cases of a cluster caught in a state of deep core collapse.

In a similar way, Sosin (1997) investigated the presence of a possible cusp in M30. Also from star counts on images obtained by HST/FOC with filters F430W $\equiv$ FOC $B$ and F480LP $\equiv$ FOC $V$ with a pixel size $0.014^{\prime \prime} / \mathrm{pix}$, he determined logarithmic cusp slopes $d \log \sigma / d \log r-0.76 \pm 0.07$, between $0.3^{\prime \prime}$ and $12^{\prime \prime}$, for stars with mass $0.69 \leq M \leq 0.76 M_{\odot}$, and $d \log \sigma / d \log r-0.82 \pm 0.11$, between $2.0^{\prime \prime}$ and $12^{\prime \prime}$, for stars with mass $0.57 \leq M \leq 0.69 M_{\odot}$. He set an upper limit of $1.9^{\prime \prime}$ on the size of any possible constant-surface-density core. 
For both clusters, all slope values are consistent with the cusp models (Cohn 1985; Chernoff \& Weinberg 1990) and with black-hole models (Bahcall \& Wolf 1977).

\section{Mass Segregation (G. Meylan)}

Mass segregation between the heavy and light stars of a dynamical system may be expected, from a theoretical point of view, since relaxation is the first dynamical process which does differentiate stars according to their mass. It produces a tendency towards equipartition of kinetic energy. The larger-mass stars involved in gravitational encounters tend to lose kinetic energy, and then fall deeper into the potential well of the cluster, while, at the same time, lower-mass stars are driven out: the stars are segregated by mass.

From an observational point of view, mass segregation has been difficult to secure from the ground. See, e.g., Bolte (1989) in the case of M30. It is only with HST observations that the case has been not only qualitatively but also quantitatively solved. See, e.g., Paresce et al. (1995) in 47 Tuc, King et al. (1995) in NGC 6397, and Ferraro et al. (1997) in NGC 6752.

Two of the best such studies have been published recently. First, Sosin \& King (1997) have investigated the structure of M15. Their mass functions (MFs) at $r=20^{\prime \prime}$ and at $r=5^{\prime}$ from the cluster center clearly show substantial mass segregation for all stars with masses between 0.55 and $0.80 M_{\odot}$.

- The MF at $r=20^{\prime \prime}$ is best fit by a power-law with slope $x=-0.75 \pm 0.26$, and

- the MF at $r=5^{\prime}$ is best fit by a power-law with slope $x=+1.00 \pm 0.25$.

The two MFs are different at the 5- $\sigma$ level. The implied mass segregation is somewhat less than predicted by a King-Michie model but somewhat greater than predicted by a Fokker-Planck model. Second, Sosin (1997) has investigated the structure of M30. His MFs at $r=0-6^{\prime \prime}, r=6-12^{\prime \prime}$, and at $r=4.6^{\prime}$ from the cluster center clearly show substantial mass segregation for all stars between 0.50 and $0.76 M_{\odot}$.

- The MF at $0^{\prime \prime}<r<6^{\prime \prime}$ is best fit by a power-law with slope $x=-3.1 \pm 0.5$,

- the MF at $6^{\prime \prime}<r<12^{\prime \prime}$ is best fit by a power-law with slope $x=-3.8 \pm 0.7$, and

- the MF at $r=4.6^{\prime}$ is best fit by a power-law with slope $x=+1.9 \pm 0.6$.

The inner and outer MFs are different at the $6-\sigma$ level. The observed degree of mass segregation matches the predictions of an isotropic multimass King model.

\section{Tidal Tails from Wide Field Imaging (G. Meylan)}

Numerical simulations (e.g., Oh \& Lin 1992, Johnstone 1993, Leon, Combes \& Leeuwin 1995) have demonstrated that continual two-body relaxation within GCs, combined with weak tidal encounters between GCs and the galactic disk and/or bulge, will lead to the development of both halos of unbound stars and tidal tails.

The observation of such halos and tidal tails has been pioneered by Grillmair et al. (1995) in the case of 12 galactic GCs, and by Grillmair et al. (1996) in the case of 4 M31 GCs. They made starcount analyses from two-color photometry (from photographic plates for the galactic GCs and from HST-WFPC2 images for the M31 GCs) in order to select and count stars with color and magnitudes consistent with the specific sequences of the cluster color-magnitude diagrams. Most of their sample clusters show extra-tidal wings in their surface density profiles and in their two-dimensional surface density maps.

Recently, Leon et al. (1997) have applied such a star-count analysis to deep $B$ and $R$ ESO Schmidt plates, $5^{\circ}$ by $5^{\circ}$ in size and centered on $\omega$ Centauri. They performed a wavelet transform of the final map in order to detect and enhance any large and weak extensions, such as tidal tails. Reconstruction of the star-count map is obtained after filtering of wavelet planes. Two strong tidal tails (Figure 1) are clearly visible towards the North-West and South-East directions. They are significant at more than the 6- $\sigma$ level.

These two tidal tails around $\omega$ Centauri are observed between 30 and $150^{\prime}$. Estimates of the stellar mass contained in these tails give values of the order 1-2\% of the total cluster mass, considered to be about $510^{6} M_{\odot}$.

Further numerical works are under way (Grillmair et al. 1997, Leon et al. 1997) to incorporate the effects of heating by the galactic disk, to study the consequences of using realistic stellar mass 


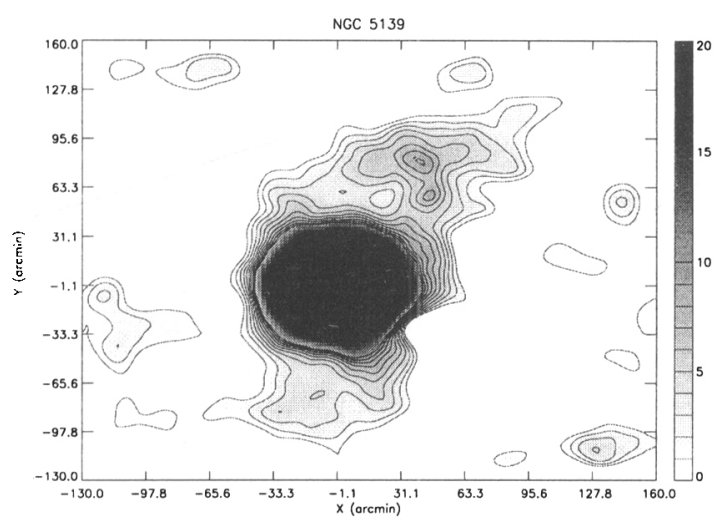

Figure 1. Tidal tails observed in the outer parts of $\omega$ Centauri by Leon et al. (1997)

distributions, and to better address the survival statistics of globular clusters in the face of continual weak tidal encounters.

\section{Signs of Tides in the Global Kinematics of Globular Clusters (G.A. Drukier)}

A globular cluster does not exist in isolation. As a component of a galaxy, a globular cluster's evolution is affected by its host galaxy. This takes two forms. First, stars are stripped from the cluster by the galactic tidal field. Second, there is a dynamical heating of the cluster due to tidal shocks as the cluster passing through the disk or near to the bulge. Observationally, the former gives rise to an unbound population of stars surrounding the globular cluster. Such excesses have been observed as discussed above, but it has not been shown that the stars are indeed unbound. To demonstrate that, kinematic data are needed, but even with such data we would need to know the total mass of the cluster. Tidal heating acts on the cluster in a more complicated manner, but the expected signature is an increase in the velocity dispersion above that expected for an isolated cluster (Allen \& Richstone 1988).

Clearly, what are needed are radial velocity measurements in the outer parts of globular clusters in order to investigate these effects. Here I report on some initial results of just such an effort at Indiana University, led by $\mathrm{H}$. Cohn and P. Lugger. We are taking advantage of the Hydra, fiberfed, multi-object spectrograph on the $3.5 \mathrm{~m}$ Wisconsin-Indiana-Yale-NOAO (WIYN) telescope to systematically measure the radial velocities of hundreds of stars in several globular clusters. Here I will discuss our results in the clusters M15 and M92.

Hydra allows for the placement of nearly 100 optical fibers in a 1 degree field. The fiber placement can be changed during the night. Fibers cannot be placed closer than $36^{\prime \prime}$, so we are generally excluded from the central half arc minute of a cluster, but the instrument is ideal for observing many stars in the outer parts of a cluster. Our strategy has been to select stars on the giant branch of the cluster and to take high resolution echelle spectra in the vicinity of the $\mathrm{Mg} \mathrm{b}$ triplet. We then use cross-correlation techniques Tonry \& Davis (1979) to measure the radial velocity. The template used is a sum of the spectra of one cluster star observed in every observing configuration. A second standard is observed to monitor the velocity consistency of the template. The zero-point is established from exposures of the twilight sky. Our median errors for member stars in M15 and M92 are $0.30 \mathrm{~km} \mathrm{~s}^{-1}$ and $0.34 \mathrm{~km} \mathrm{~s}^{-1}$, respectively.

Candidate selection, as mentioned, is on the basis of photometry. We have managed to increase our efficiency in observing members by selecting stars in the C-M vs. M-T2 color-color diagram in the Washington system Geisler etal. (1991). Repeat observations were made of most of the members, with membership being determined on the basis of velocity consistency and strength of the $\mathrm{Mg} b$ lines. The last are a good luminosity indicator and allow us to reject field dwarfs with velocities similar to the cluster. In total we have observed nearly 600 stars in each of M15 and 

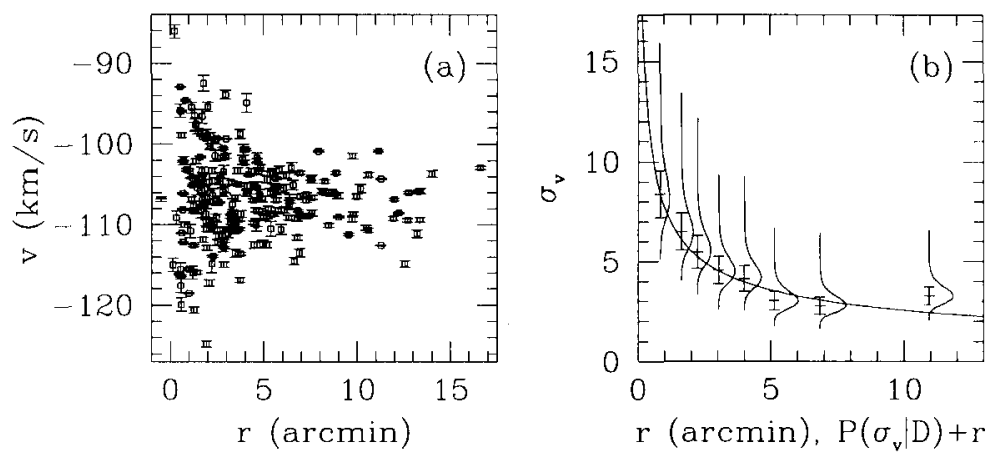

Figure 2. (a) Heliocentric velocities for the member stars in M15. The dispersion appears to increase again outside of $7^{\prime}$. (b) Velocity dispersion profile based on the velocities at left. The curves at each radius give the relative probability of the velocity dispersion at that radius having the value on the ordinate. The points show the mode and the boundary of the symmetric region containing $68.5 \%$ of the probability. The smooth curve is a power-law fit to the velocities inside of $8^{\prime}$

M92, detecting 230 members in the former and 304 in the latter. The higher number of members detected in M92 reflects the use of Washington photometry in candidate selection for this cluster.

Figure 2a shows the velocities of the member stars in M15. What is striking in this diagram is that after decreasing outwards, the velocity dispersion appears to increase again outside of $7^{\prime}$ from the cluster center. In order to characterize this, we have developed a new analysis method based on Bayesian techniques. With this method we can derive probability distributions for the velocity dispersion and discuss the relative probability of different models for the velocity dispersion profile.

In Figure $2 b$ we show the results for M15. Our first model bins the data and assumes a constant value for the velocity dispersion across the bin. The curves at each radius in Figure $2 b$ show the probability distribution for the velocity dispersion in each bin. The model show also allows for the cluster to be rotating. We find a small amount of rotation, mostly in the bin at $4^{\prime}$, and the model with rotation is mildly favored over one without. Comparison of the last two bins gives odds of 3.3 to 1 that the velocity dispersion in the last bin is larger than in the penultimate bin. A stronger claim can be made by our second model, shown as the smooth curve in Figure 2b. Here we fit all the velocities with $r<8^{\prime}$ with a power law form for the velocity dispersion profile and then compare the extrapolated value with the observed velocities for the 31 stars with $r>8^{\prime}$. The odds are 320 to 1 against the velocities of these stars being consistent with the extension of the power law. This is roughly a 2.7- $\sigma$ result. Similar behavior is seen in our M92 data, but the size of the effect depends on whether a few stars in the outer part of the cluster with relatively high velocities with respect to the mean are deemed members are not. The analysis of the more recent M92 data is still underway to resolve this question.

Our data in M15 and M92 strongly suggest that the expected heating from the Galaxy is occuring. It could be argued that the increase in the dispersion of the radial velocity is due to strong tangential anisotropy in the outer parts of these clusters rather than an increase in the overall velocity dispersion. This is unlikely to be the case, since dynamical models for the evolution of isolated globular clusters clearly show that they are strongly radially anisotropic (Larson 1970, Spitzer \& Shull 1975, Cohn 1979). Converting these radial orbits to circular ones at the same apocenter requires energy input. Thus, whatever the anisotropy in the outer part of the clusters, tidal heating is required.

Grillmair et al. (1995) claim that the tidal radius of M15 is $23^{\prime}$. The simulations of (Allen \& Richstone 1988) suggest that the kinematic signature of the tidal radius is a minimum in the velocity dispersion profile. On this basis, the tidal radius of M15 is around $8^{\prime}$. It is not clear that these two definitions of the tidal radius are referring to the same thing, so the apparent discrepancy needs to be taken with caution. Further, new studies with improved treatments of tidal heating have been done (e.g. Kundic \& Ostriker 1995). Unfortunately, these have not presented projected 
velocity dispersion profiles, and so we are unable to compare our observations with them. It is to be hoped that future theoretical studies will take into account supporting observations and present velocity dispersion profiles. Full details of these observations are in Drukier et al. (1998a,b).

\section{Acknowledgement}

GM would like to thank his collaborators, T. Bridges, F. Combes, S. G. Djorgovski, P. Jablonka, S. Leon, and A. Sarajedini, for allowing the presentation of results in advance of publication.

\section{References}

Allen A.J., Richstone D.O., 1988, ApJ, 325, 583

Ambartsumian V.A., 1938, Ann. Leningrad State Univ., 22, 19

Bahcall J.N., Wolf R.A., 1977, ApJ, 216, 883

Bailey S.I. 1893, Astronomy and Astro-Physics, XII, 689

Binney J., Tremaine S., 1987, Galactic Dynamics, (Princeton: Princeton University Press)

Bolte M., 1989, ApJ J 341, 168

Chandrasekhar S., 1942, Principles of Stellar Dynamics, (Chicago: Univ. of Chicago Press)

Chernoff D.F., Weinberg M.D., 1990, ApJ, 351, 121

Cohn H., 1979, ApJ, 234, 1036

Cohn H., 1985, in Dynamics of Star Clusters, IAU Symp. 113, eds. Goodman J. \& Hut P., (Dordrecht: Reidel), p. 161

Dickens R.J., Woolley R., 1967, Royal Obs. Bull. \# 128

Djorgovski S.G., et al., 1997, ApJ, 474, L19

Drukier G.A., Slavin S.D., Cohn H.N., Lugger P.M., Berrington R.C., Murphy B.W., Seitzer P.O., 1998a, AJ, submitted

Drukier G.A. et al. 1998b, in preparation

Eddington A.S., 1916, MNRAS, 76, 572 (and references therein)

Ferraro F.R., et al., 1997, MNRAS, 286, 1012

Geisler D., Clariá J.J., Minniti D., 1991, AJ, 102, 1836

Grillmair C.J., Freeman K.C., Irwin M., Quinn P.J., 1995, AJ, 109, 2553

Grillmair C.J., et al., 1996, AJ, 111, 2293

Grillmair et al,, 1997, in prep.

Gunn J.E., Griffin R.F., 1979, AJ, 84, 752

Hénon M., 1961, Ann. d'Astrophys., 24, 369

Illingworth G., 1976, AJ, 204, 73

Jablonka P., Sarajedini A., Bridges T., Meylan G., 1998, in prep.

Jeans J.H., 1916, MNRAS, 76, 567 (and references therein)

Johnstone D., 1993, AJ, 105, 155

King I.R., 1966, AJ, 71, 64 (and references therein)

King I.R., Sosin C., Cool A.M., 1995, ApJ, 452, L33

Kormendy J., 1985, ApJ, 295, 73

Kundic̀ T., Ostriker J.P. 1995, ApJ, 438, 702

Larson R.B., 1970, MNRAS, 150, 93

Leon S., Combes F., Leeuwin F., 1995, in "Dynamics of the Galaxy", eds. Alfaro E. \& Delgado D.M., (Cambridge: CUP).

Leon S., et al., 1998, in prep. (see also a poster in these proceedings)

Lynden-Bell D., Wood R., 1968, MNRAS, 138, 495

Makino J., 1996, ApJ, 471, 796

Mayor M., Meylan G., Udry S., et al., 1997, AJ, 114, 1087

Merritt D., 1996, AJ, 112, 1085

Merritt D., Meylan G., Mayor M., 1997, AJ, 114, 1074

Meylan G., 1987, A\&A, 184, 144

Meylan G., Hegggie D.C., 1997, A\&AR, 8, 1

Meylan G., Jablonka P., Djorgovski, S.G., Sarajedini A., Bridges T., Rich R.M., 1998, in prep.

Meylan G., Mayor M., Duquennoy A., Dubath P., 1995, A\&A, 303, 761

Michie R.W., 1963, MNRAS, 126, 499

Oh K.S., Lin D.N.C., 1992, ApJ, 386, 519

Oort J.H., van Herk, G., 1959, Bull. Astron. Inst. Neth., 14, 299

Paresce F., De Marchi G., Jedrzejewski R., 1995, ApJ, 442, L57

Pickering E.C., 1897, Ann. Harvard Coll. Obs., 26, 213

Plummer H.C., 1911, MNRAS, 71, 460

Sosin C., A.J, 1997, in press

Sosin C., King I.R, 1997, AJ, 113, 1328

Spitzer L., 1940, MNRAS, 100, 396

Spitzer L., Shull J.M., 1975, ApJ, 200, 339

Tonry J., Davis M., 1979, AJ, 84, 1511

von Hoerner S., 1957, ApJ, 125, 451 